\title{
Control de la placa dental en pacientes con ortodoncia. Una revisión de la literatura
}

\author{
Control of the dental plate in patients with orthodontics. A review of literature \\ Nelly Liliana Sánchez Vásquez ${ }^{1 a}$
}

\section{RESUMEN}

Conforme los tratamientos ortodonticos han ido incrementándose también han ido en aumento las patologias producto del mal control de placa durante el tratamiento se ha observado un considerable incremento en la aparición de caries o manchas blancas después de los tratamientos ortodonticos asi como la enfermedad periodontal. El control de placa es sumamente importante puesto que al presentar aditamentos ortodonticos disminuye la capacidad autolimpieza asi como se incrementan las zonas retentivas y propensas a la formación del biofilm. Para evitar que los pacientes presenten este problema se decide hacer una revisión bibliografica y asi plantear una alternativa para el clínico I

Palabras clave: Placa Dental; Ortodoncia; Ionomero ; Higiene Bucal. (Fuente: DeCS BIREME)

\section{ABSTRACT}

As the orthodontic treatments have been increasing, the pathologies resulting from poor plaque control have also increased while the laps of the treatment ; There is a considerable increase in the appearance of caries or white spots after orthodontic treatments as well as periodontal disease. The control of plaque is extremely important since when presenting orthodontic attachments we diminish the capacity self-cleaning as well as we increase the retentive zones and prone to the formation of the biofilm. To prevent patients from presenting this problem, it is decided to make a bibliographic review and thus propose an alternative for the clinician.

Key words: Dental plaque; Orthodontics; Ionomer ;Oral hygiene. (Source: MeSH NLM)

${ }^{1}$ Seguro Social de Salud del Perú (EsSALUD) adontólogo

Correspondencia:

Nelly Liliana Sánchez Vásquez

Correo electrónico: dranelly.sanchez@gmail.com
Este es un artículo Open Access distribuido bajo la licencia Creative Commons Atribución-NoComercial- Compartir Igual 4.0

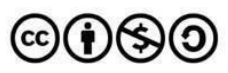

Citar como: Sánchez Vásquez N. Control de la placa dental en pacientes con ortodoncia. Una revisión de la literatura. KIRU. 2019 abr-jun; 16(2): 92-96. https://doi.org/10.24265/kiru.2019.v16n2.06 


\section{INTRODUCCIÓN}

En los últimos años las consideraciones estéticas han cobrado mayor importancia por lo que ha llevado a una disminución de costos tanto en tratamientos de estética como de ortodoncia; adicionalmente hay un mayor número de profesionales entrenados lo que brinda más opciones de atención para los pacientes ${ }^{(1-4)}$.

Uno de los tratamientos más utilizados es el uso de brackets que en promedio dura aproximadamente dos años, esta condición favorece la retención de placa dental y obliga al paciente a esmerar su cuidado bucal a fin de prevenir caries dental o enfermedad periodontal ${ }^{(1,5)}$. Un adecuado control de placa, aplicando la técnica de cepillado dental correcta puede ayudar a prevenir la aparición temprana de la caries o enfermedad periodontal, sin embargo, el tipo de aditamento utilizado en los pacientes, el acercamiento que tenga el paciente con su profesional, también es importante ${ }^{(6,10)}$.

Frecuentemente después del tratamiento ortodontico se presentan caries y enfermedad periodontal $^{(1,2,5,9)}$. Los casos de enfermedad periodontal pueden exacerbarse durante el tratamiento $(2,8,19)$.

Las ligaduras utilizadas en ortodoncia, pueden provocar una alta retención de biofilm lo que puede generar el inicio de la enfermedad periodontal.1,9 Una inadecuada técnica de colocación de dispositivos orotdonticos como bandas o ligas y el tipo de saliva del paciente pueden ser desfavorables para el buen control de biofilm ${ }^{(3-7)}$.

El propósito de esta revisión fue conocer a través de la búsqueda bibliográfica la relación existente materiales usados en ortodoncia y su implicancia con respecto a la retención de placa.

En un metaanálisis realizado por Yang et al ${ }^{(9)}$ sobre el uso de brackets autoligantes y convencionales concluyeron que los autoligantes no son mejores que los convencionales en cuanto a mantener una mejor higiene bucal, también señalan que la agregación bacteriana, el índice de placa y las condiciones periodontales, pueden modificar el impacto del control del biofilm.9 También influye en las condiciones periodontales del paciente cuando buscando estética o funcionalidad no se respetan aspectos fundamentales como los espacios biológicos ${ }^{(1-6)}$.

\section{Relación existente aditamentos usados en ortodoncia y su implicancia con respecto a la retención de placa}

Diversos estudios reportan la mayor incidencia en enfermedad periodontal y caries en pacientes que han recibido tratamiento ortodóncico debido a la relación entre acumulación de biofilm y ligas o brackets ${ }^{(1-2)}$. Estudios realizados por Sawhney et al1 refiere que la porfiromona gingivalis coloniza estos aditamentos ortodóncicos. Baracaldo et al 6 concluyó que la técnica de cepillado con mejor eficiencia es la técnica vertical en pacientes con ortodoncia fija. Así mismo se sabe que el control mecánico más el químico, así como la instrucción y motivación en higiene bucal realizada al paciente garantiza minimizar las descalificaciones y el sangrado gingival. Por ello es importante primero identificar el riesgo de caries y enfermedad periodontal para aplicar una terapia profiláctica adecuada.

Migliori et al 2 plantean un esquema que se basa principalmente en determinar el riesgo paciente. Se evalúa la presencia de placa con un índice de Sillnes y Loe, motivando e instruyendo al paciente en higiene bucal y se le explica las posibles complicaciones de no seguir las indicaciones, según Thomson et.all2 esta explicación debe ser visual y $\operatorname{escrita}^{(12,13)}$. Posteriormente reciben su control profesional en higiene bucal con evidenciadores seguida de una buena profilaxis y se le coloca preventivamente su topicación de flúor. Este procedimiento se plantea a los pacientes preventivamente un mes antes de colocación de las bandas brackets y ligas propias del tratamiento ${ }^{(1,9)}$.

En la cita de instalación se realiza un examen de conocimiento básicos, un índice de Sillnes y Loe y una demostración del uso del cepillo eléctrico. Tres meses después se programa otra cita, para un nuevo índice de higiene oral, remoción del arco, realizar la profilaxis la fluorización y evaluación de las complicaciones. Según los resultados de los índices, si el índice es menor a tres se le indica como paciente de bajo riesgo y su control será cada seis meses ; Mayor o igual a tres será un paciente de riesgo moderado y los controles serán cada tres meses y de tener mayor a tres significa que adicionalmente tiene zonas de desmineralización y se citará al paciente cada tres meses y se aplicará el calcio fosfato péptido caseína como parte del protocolo para prevenir a la caries y enfermedad periodontal. Este control con el higienista dental se realiza simultáneamente al control mensual del ortodoncista quien evaluará los factores de riesgo. 
Este proceso se realizará durante todo el tiempo que dure el tratamiento ortodoncico.

\section{Materiales en ortodoncia}

Yang y col9 determinaron que no existen diferencias significativas entre el uso de autoligantes e versus brackets convencionales higiene dental. Sawhney y col1 en su estudio demostró que las ligaduras utilizadas en el tratamiento ortodóncico eran la principal causa de colonización microbiana.

Swahney et al 1, reportaron que las principales fuerzas que intervienen en la adhesión son las de Vander Walls, las de adhesión a las lectinas y las interacciones electrostáticas. Además que los aparatos ortodóncicos disminuyen el efecto de los mecanismos de autolimpieza de la saliva, la lengua y las mejillas y que estos a su vez favorecen la retención de biofilm y aumento de zonas retentivas, lo que representa un conjunto de factores que de no ser controlados afectaran la mucosa oral y aumentara el riesgo de caries ${ }^{(14,15)}$.

En un estudio realizado por Marsh et al14 observaron que los pacientes portadores de los aparatos de ortodoncia presentaban aumento del flujo salival del $\mathrm{pH}$ salival, y niveles bacterianos 15. Otros factores que condicionan el aumento el biofilm, son la maloclusión, mala higiene oral y una dieta cariogénica ${ }^{(1,2,14,15,16)}$. Los aparatos de ortodoncia aumentan las áreas retentivas y aumentan la cantidad de nichos bacterianos. Se observó caries temprana, desmineralización y lesiones de mancha blanca en pacientes con tratamiento de ortodoncia e higiene bucal inadecuada, situación que se agravo en casos de pacientes inmunosuprimidos o con enfermedades sistémica ${ }^{(9,14,15,16)}$.

Estudios microbiológicos realizados con reacción de cademna polimerasa (PCR) mostraron la presencia de Prevotella intermedia, Prevotella nigrescen, Porphyromona gingivallis, Tannerella forsythia, Agregatibacter Actinomicetemcomitans ${ }^{(9,10,11,12,13,15)}$.

Uno de los factores contribuyentes al aumento del biofilm, adicionalmente a los brackets y bandas, son los cementos utilizados para la fijación ${ }^{(17)}$.

En un estudio realizado por Wang et al 10 determinaron que el uso de ionómeros modificados para cementación daban mejores resultados en la prevención de la aparición de la mancha blanca que las resinas convencionales y esta se debía principalmente a la presencia del flúor $(18,19,20,21,26)$, sin embargo este efecto anticariógeno es de muy corta duración ${ }^{(21,22,23,24)}$.
Wang et al 10 describen a la plata como el metal que tiene un efecto antibacteriano, además de un efecto antifungico y antiviral. y concluyendo que la acción que estos iones de plata activos en el ionomero inhiben la enzima encargada de la replicación bacteriana presente en el ADN bloqueando inicio de la formación de la placa. Por otro lado, se describe en estudios a seis meses, que este ionomero con iones de NAg inhibe el crecimiento del Estreptococos mutans sobre el ionomero y además no producen cambios en la adhesión, en la coloración, ni aumenta la rugosidad (25 26 27).

Un estudio reporta una disminución en el crecimiento bacteriano de los adhesivos cuya composición incluyan oxido de cobre en nanopartículas pero aún está en proceso de investigación ${ }^{(11)}$.

\section{Innovaciones en el control de placa}

Erbe et al. refiere en un trabajo sobre adolecentes, el uso de cepillos manuales versus cepillos interactivos eléctricos que en ambos grupos de estudio se logró un control eficiente de placa sin embargo con el uso de cepillos interactivos los adolescentes alcanzaron mejores resultados debido a que este sistema permite controlar frecuencia intensidad duración y modo en tiempo real ${ }^{(12,4,27)}$.

Scheerman et al 27en un estudio randomizado explican la aplicación de los Smart phones para el control de placa en pacientes con ortodoncia fija. Ellos crearon una app y la evaluaron por 12 semanas en adolescentes. Dividieron el estudio en dos fases una motivacional en donde se les indicó la frecuencia, tipo de cepillo, tiempo de cepillado, índice de higiene oral los 5 pasos del cepillado, así como el uso de enjuagues con flúor o clorhexidina. La segunda fase era del control mediante una app, en donde los adolescentes ingresaron sus datos como tipo de cepillo, frecuencia de cepillado, uso de enjuagues y una foto tipo selfie que el paciente envíaba de sus dientes a través de la app, previa aplicación de revelador de placa. La aplicación enviaba el promedio alcanzado por el paciente así como un plan de higiene que debería ser respetado. Complementaron con videos de instrucción cortos para reforzar lo instruido sobre el cepillado, tiempo, y aditamentos de higiene. Al finalizar la semana el adolescente debía enviar un selfie de sus dientes para ser evaluado. Concluyeron que los adolescentes evaluados se sintieron satisfechos y reconocieron la importancia del uso de este dispositivo y observaron un control eficiente de placa. 
Se ha evidenciado que las bacterias se adhieren a las ligas y brackets ortodónticos sin diferencias significativas entre brackets autoligantes y convencionales. La principal bacteria es la porfiromona gingivalis.

La técnica de cepillado con mejores resultados es la técnica vertical sin embargo el uso del cepillo eléctrico es el que tiene mejores resultados siendo este el más recomendado en pacientes con tratamiento ortodóntico

También se registra buen control de la placa en pacientes que siguen un esquema especifico de control caracterizado por el uso de evidenciadores y controles periódicos un mes antes de la adhesión y luego cada seis meses en pacientes sanos no obstante en pacientes con índices de placa mayores a tres o igual estos controles serán cada tres meses y si presentara zonas de descalcificación se adicionaría el uso del fosfato péptido de caseína.

En un estudio realizado por Wang y cols se concluyó que existe una ventaja con el uso de ionomeros en relación con la resina en lo que corresponde a prevención en la aparición de la mancha blanca, y rescata el uso de ionomeros reforzados con plata como materiales de cementación con características antifúngicas y antibacterial .inhibiendo la enzima de la replicación bacteriana.

Desde que aparecieron las App y los móviles inteligentes miles de usos se les han dado y el control de placa no ha sido la excepción .En la actualidad existen App que mediante el uso del bluetooth permite manejar intensidad revoluciones o torque de los cepillos dependiendo de la evaluación hecha con evidenciadores de placa que el propio paciente coloca sobre sus dientes y que mediante un selfie es enviada al clínico.

Es evidente que la odontología en lo que es el control de placa no es ajena a los avances tecnológicos pero sería importante realizar mayor número de investigaciones en estos aspectos.

\section{Conclusiones}

En la revisión bibliográfica se encontró evidencia significativa entre la caries y enfermedad periodontal y la ausencia de control de placa en pacientes con tratamiento ortodontico.

Los factores que contribuyen al control del biofilm en los pacientes con tratamiento de ortodoncia son: el uso de cementos de ion omero preferentemente reforzados con plata para la cementación de bandas y brackets; uso de cepillos eléctricos sino se pudiera usar los manuales con la técnica vertical.
Se recomienda realizar un control de placa profesional cada tres meses si presentara lesiones, en zonas de descalcificación utilizar el calcio fosfato caseína. El protocolo de trabajo con pacientes en tratamiento ortodontico debe de ser multidisciplinario periodoncistas y ortodoncistas estar en constante comunicación

Participación de autoría: NS reviso la literatura, redactó y aprobó el artículo.

Conflicto de interés. El autor declaró no tener conflictos de interés

Fuente de financiamiento: Autofinanciado

\section{REFERENCIAS}

1. Sawhney R, Sharma R , Sharma K . Microbial Colonization on Elastomeric Ligatures during Orthodontic Therapeutics: An Overview. Turk J Orthod. $2018 ; 31(1): 21-25$.

2. Migliorati M, Isaia L, Cassaro A, Rivetti A, Silvestrini-Biavati $F$ ,Gastaldo $L$ et al . Efficacy of professional hygiene and prophylaxis on preventing plaque increase in orthodontic patients with multibracket applicances: a systematic review. Eur J Orthod. 2015 ; 37(3):297-307.

3. Scribante A, Gandini P , Sfondrini MF , CollesanoV ,Tovt G, Bernardinelli L . Dental Hygiene and Orthodontics: Effect of Ultrasonic Instrumentation on Bonding Efficacy of Different Lingual Orthodontic Brackets.Biomed Res Int. 2017:

4. Zhang N, Zhang $\mathrm{K}$, Weir M, Xu D, Hocki $\mathrm{H}$ et al.Effects of water-aging for 6 months on the durability of novel antimicrobial and protein-repellent dental bonding agent. Int Oral Sci.2018 Jun; 10(2): 18.

5. Alavi S, Yaraghi $\mathrm{N}$. The effect of fluoride varnish and clorhexidine gel on white spot and gingival and plaque indices in fixed orthodontic patients: A placebo-controlled study.Dent Res J(Isfanhan). 2018 ;15(4):276-282.

6. Baracaldo Y,Alexander F, Cabuya C, Hurtado R, Ricaurte E , Gamboa D , Báez L. Evaluación de las técnicas de cepillado de bass y vertical en un grupo de pacientes en tratamiento de ortodoncia con técnica MBT. Acta Odontol. Colomb.2012., 2(2):33-43

7. Pan S, Liu $Y$, Prevalence of fimA genotypes of Porphyromonas gingivalis in adolescent orthodontic patients PLoS One. 2017; 12(11).

8. Lu H, Tang $\mathrm{H}$, Zhou $\mathrm{T}$. Assement of periodontal health status in patients undergoing orthodontic treatment with fixed appliacances and Invisaling system: A meta-analysis. Medicine .2018; 97:e 0248.

9. Yang X, Su N,Shi Z. Effects of self-ligating brackets on oral hygiene and discomfort: a systematic review and metaanalysis of randomized controlled clinical trials. Int $\mathrm{J}$ Dent Hygiene 15,2017;16-22

10. Wang $X$, Wang B, Wang Y. Antibacterial orthodontic cement to combat biofilm and white spot lesions.Am $\mathrm{J}$ of Orthod Dentofacial Othop: 2015 ; 148; 974-81. 
11. Toodehzaeim M, Zandi $\mathrm{H}$, Meshkani M ,Firouzabadi A.The Effect of $\mathrm{CuO}$ Nanoparticles on Antimicrobial Effects and Shear Bond Strength of Orth

12. Thomson, A.M., Cunningham, S.J. and Hunt, N. A comparison of information retention at an initial orthodontic consultation. Eur J Orthod.2001, 23, 169178.

13. Kitada K, Toledo A, Oho T. Increase in detectable opportunistic bacteria in oral cavity of orthodontic patients. Int J Dent Hyg 2009; 7: 121-5. odontic Adhesives J Dent (Shiraz). 2018;19(1): 1-5.

14. Marsh PD, Moter A, Devine DA. Dental plaque biofilms: communities, conflict and control. Periodontol 2000 2011; 55: 16-35.

15. Lara-Carrillo E, Montiel-Bastida NM, Sánchez-Pérez L, Alanis-Tavira J. Effect of orthodontic treatment on saliva, plaque and the levels of Streptococcus mutans and Lactobacillus. Med Oral Patol Oral Cir Bucal 2010; 15: e924-9

16. Paschos $\mathrm{E}$, Kleinschrodt $\mathrm{T}$, Clementino-Luedemann $\mathrm{T}$, Huth KC, Hickel R, Kunzelmann $\mathrm{KH}$, et al. Effect of different bonding agents on prevention of enamel demineralization around orthodontic brackets. Am J Orthod Dentofacial Orthop 2009;135:603-12.

17. Tuncer C, Tuncer BB, Ulusoy C. Effect of fluoridereleasing lightcured resin on shear bond strength of orthodontic brackets. Am J Orthod Dentofacial Orthop 2009;135:14.e1-6.

18. Badawi H, Evans RD, Wilson M, Ready D, Noar JH, Pratten J. The effect of orthodontic bonding materials on dental plaque accumulation and composition in vitro. Biomaterials 2003;24: 3345-50.

19. Enaia M, Bock N, Ruf S. White-spot lesions during multibracket appliance treatment: a challenge for clinical excellence. Am J Orthod Dentofacial Orthop 2011;140:e17-2
20. Guzman-Armstrong S, Chalmers J, Warren JJ. White spot lesions: prevention and treatment. Am J Orthod Dentofacial Orthop 2010; 138:690-6

21. Uysal T, Amasyali M, Ozcan S, Koyuturk AE, Sagdic D. Effect of antibacterial monomer-containing adhesive on enamel demineralization around orthodontic brackets: an in-vivo study. Am J Orthod Dentofacial Orthop 2011;139:650-6.

22. Rogers S, Chadwick B, Treasure E. Fluoride-containing orthodontic adhesives and decalcification in patients with fixed appliances:a systematic review. Am J Orthod Dentofacial Orthop 2010;138: 390.e1-8

23. Yoshida K, Tanagawa M, Atsuta M. Characterization and inhibitory effect of antibacterial dental resin composites incorporating silver-supported materials. J Biomed Mater Res 1999; 47:516-22.

24. Zhang K, Cheng L, Wu EJ, Weir MD, Bai Y, Xu HH. Effect of wateraging on dentin bond strength and antibiofilm activity of bonding agent containing antibacterial monomer dimethylaminododecyl methacrylate. J Dent 2013;41:504-13.

25. Reynolds IR. A review of direct orthodontic bonding. $\mathrm{Br}$ J Orthod 1975;2:171-8.

26. Erbe C, Cunningham P,Farrell S, A comparative asseessment of plaque removal and toothbrush among adolescents: a single-center, single-blind randomized controlled trial.BMC Oral Health.2018; 18: 130

27. Scheerman J, Meijel B, Empelen P, Gem J, Kramer C, Gijsbert H. W. Verrips, Pakpour A, Matheus C, Van den Braak T, and Van Loveren C. Study protocol of a randomized controlled trial to test the effect of a smartphone application on oral-health behavior and oral hygiene in adolescents with fixed orthodontic appliances BMC Oral Health. 2018; 18: 19 